\title{
ON LOWER BOUNDS OF THE NATURAL FREQUENCIES OF INHOMOGENEOUS PLATES*
}

\author{
$\mathrm{BY}$ \\ V. KOMKOV \\ Texas Tech University, Lubbock
}

1. Introduction. This note deals with an application of the intermediate problem technique to the computation of the natural frequencies of a vibrating inhomogeneous plate. The intermediate problem technique was first introduced in 1935 by Alexander Weinstein [13]. An abstract formulation of this technique was developed by N. Aronszajn [1], [2], and is related to the theories of N. Bazley and D. W. Fox [4], [5], [6], N. Aronszajn [3] and to further developments of A. Weinstein in [14], [15], [16], G. Fichera [8], [9], J. B. Diaz [7], T. Kato [11], and S. T. Kuroda [12]. An expository article of A. Weinstein [17] and a monograph of S. H. Gould [10] provide a review of this theory. This note uses a method closely related to the original technique of Weinstein to replace the problem of finding the lower bounds to the natural frequencies of a vibrating inhomogeneous plate subject to fairly complex boundary conditions by the much easier problem of determining the natural frequencies of the inhomogeneous membrane which is freely supported on the boundary.

In particular we formulate the Weinstein determinant for the plate problem in terms of the eigenfunctions and eigenvalues of the corresponding membrane problem.

2.0 Physical assumptions and notation. We assume the correctness of Hooke's law and the usual linear hypothesis of thin plate theory. The plate occupies a finitely connected compact subset $\bar{\Omega}$ of the Euclidean plane $E^{2}$. The boundary $\partial \Omega$ of $\bar{\Omega}$ consists of a finite number of straight lines, whose union will be denoted by $\Gamma_{1}$, and of finite number of smooth ares, whose union will be denoted by $\Gamma_{2} \cdot \partial \bar{\Omega}=\overline{\Gamma_{1} \cup \Gamma_{2}}$ (where the bar denotes the closure operation). The interior of $\bar{\Omega}$ will be denoted by $\Omega$. $n$ will denote the unit vector in the direction of the exterior normal to the boundary $\partial \Omega$, whenever it is defined. $x, y$ are the Cartesian coordinates, $t$ is the time variable, $\nabla^{2}$ denotes the Laplacian:

$$
\nabla^{2}=\frac{\partial^{2}}{\partial x^{2}}+\frac{\partial^{2}}{\partial y^{2}} .
$$

$\diamond^{4}$ denotes the operator defined by:

$$
\diamond^{4}(A, B)=\frac{\partial^{2} A}{\partial x^{2}} \frac{\partial^{2} B}{\partial y^{2}}-2 \frac{\partial^{2} A}{\partial x \partial y} \frac{\partial^{2} B}{\partial x \partial y}+\frac{\partial^{2} A}{\partial y^{2}} \frac{\partial^{2} B}{\partial x^{2}} .
$$

\footnotetext{
* Received September 10, 1972.
} 
The physical meanings of symbols used here are: $h(x, y)$ is thickness of the plate $(h>0$ in $\Omega), \nu$ is Poisson's ration $\left(0<\nu<\frac{1}{2}\right), \rho$ is mass density of the plate, $\rho(x, y)>0$ in $\bar{\Omega}$, $E$ is Young's modulus $(E>0), D$ is flexural rigidity: $D=E h^{3} / 12\left(1-\nu^{2}\right)$, and $w(x, y, t)$ is the transverse displacement. The displacement function $w(x, y, t)$ is assumed to be twice continuously differentiable on $\bar{\Omega}$. (Note: We do not assume $w \in C^{4}(\Omega)$.)

2.1. Definitions of Hilbert spaces $L_{2}(\Omega), L_{2}(\partial \Omega), L_{2,4}(\Omega), L_{2,4}(\partial)$. We consider a class of real-valued functions of two real variables which are square integrable in the region $\Omega \subset E^{2}$, and form the space $L_{2}(\Omega)$ in the usual manner by identifying all functions which differ only on a set of measure zero in $\Omega$. For any functions $f(x, y), g(x, y) \in L_{2}(\Omega)$ the inner product

$$
\iint_{\Omega} f(x, y) \cdot g(x, y) d x d y=(f, g)_{\Omega}
$$

is defined. $\left((f, f)_{\Omega}\right)^{1 / 2}=\|f\|_{\Omega}$ is the usual $L_{2}(\Omega)$ norm of $f(x, y)$.

In a similar way we define the space $L_{2}(\partial \Omega)$ of functions $f(x, y)$ defined on $\partial \Omega$ for which the integral $\mathscr{S}_{\partial \Omega} f^{2}(x, y) d s$ exists. The inner product of $f, g \in L_{2}(\partial \Omega)$ is defined by the contour integral:

$$
(f, g)_{\partial \Omega}=\oint_{\partial \Omega} f(x, y) \cdot g(x, y) d s,
$$

and $\left((f, f)_{\partial \Omega}\right)^{1 / 2}=\|f\|_{\partial \Omega}$ defines the $L_{2}(\partial \Omega)$ norm of $f(x, y)$.

We could also consider vector-valued functions $A(x, y)=\left[A_{1}(x, y), A_{2}(x, y), A_{3}(x, y)\right.$, $A_{4}(x, y)$ ], that is vectors with four components, such that each component $A_{i}(x, y)$, $i=1,2,3,4$, belongs to $L_{2}(\Omega)$. We define $L_{2,4}(\Omega)$ as the Hilbert space of all such vectors with the inner product:

$$
\begin{aligned}
\langle\mathbf{A}, \mathbf{B}\rangle_{\Omega}= & \iint_{\Omega}\left[A_{1}(x, y) B_{1}(x, y)+A_{2}(x, y) B_{2}(x, y)+A_{3}(x, y) B_{3}(x, y)\right. \\
& \left.+A_{4}(x, y) B_{4}(x, y)\right] d x d y .
\end{aligned}
$$

The $L_{2,4}$ norm is: $\|\mathbf{A}\|=\left(\langle\mathbf{A}, \mathbf{A}\rangle_{\Omega}\right)^{1 / 2}$. The definition of $L_{2,4}(\partial \Omega)$ is analogous.

3. The basic equation and the boundary conditions. In $\Omega$ the displacement function $w(x, y)$ obeys the differential equation:

$\nabla^{2}\left[D(x, y) \nabla^{2} w(x, y, t)\right]-(1-\nu) \diamond^{4}(D(x, y), w(x, y, t))+\rho(x, y) \frac{\partial^{2} w(x, y, t)}{\partial t^{2}}=0$.

A separation of variables reduces Eq. (1) to the form of two simultaneous equations:

$$
\begin{gathered}
\nabla^{2}\left[D(x, y) \nabla^{2} w_{0}(x, y)\right]-(1-\nu) \triangleright^{4}\left(D(x, y), w_{0}(x, y)\right)-\omega^{2} \rho(x, y) w_{0}(x, y)=0 \\
w(x, y, t)=w_{0}(x, y) \exp (j \omega t) .
\end{gathered}
$$

Equation (2a) can be rewritten as:

$$
\nabla^{2}\left[D(x, y) \nabla^{2}\left(w_{0}(x, y)\right]-(1-\nu) \diamond^{4}\left(D, w_{0}\right)=\lambda \rho w_{0}(x, y) .\right.
$$

This equation may not be satisfied in the classical sense, because the physically motivated differentiability assumptions do not specify the existence of the first term of this equation 
everywhere in $\Omega$, and we may replace (2a) by its weak form:

$$
\begin{array}{r}
\iint_{\Omega}\left\{\nabla^{2}\left[D(x, y) \nabla^{2} w_{0}(x, y)\right] \cdot \psi(x, y)-(1-\nu) \triangleright^{4}\left(D(x, y), w_{0}(x, y)\right) \cdot \psi(x, y)\right\} d x d y \\
=\lambda \iint_{\Omega} \rho(x, y) w_{0}(x, y) \psi(x, y) d x d y
\end{array}
$$

for any $\psi(x, y) \in L_{2}(\Omega)$. The use of the fundamental theorem of calculus of variations gives us:

$$
\left(\left[\nabla^{2}\left(D \nabla^{2} w_{0}\right)-(1-\nu) \diamond^{4}\left(D, w_{0}\right)\right], \psi\right)_{\Omega}=\lambda\left(\rho w_{0}, \psi\right)_{\Omega} .
$$

On the $\Gamma_{1}$ part of the boundary $\partial \Omega$ the function $w_{0}(x, y)$ obeys the simple support conditions:

$$
\begin{gathered}
w_{0}(x, y)=0, \\
\nu \nabla^{2} w_{0}(x, y)+(1-\nu) \frac{\partial^{2} w_{0}(x, y)}{\partial n^{2}}=0 .
\end{gathered}
$$

On the $\Gamma_{2}$ part of the boundary the plate is clamped, i.e.:

$$
w_{0}(x, y)=0, \quad \partial w_{0}(x, y) / \partial n=0
$$

We make the following assumptions only concerning the nature of solutions $w_{0}(x, y) \in \bar{\Omega}$ :

i) $w_{0}(x, y) \in C^{2}(\bar{\Omega})$

ii) $\nabla^{2}\left(D \nabla^{2} w_{0}\right)$ is regarded as a generalized derivative in the sense of Sobolev in $\Omega$.

4. Solution of the intermediate problem for the case: $\diamond^{4}(D, w)=0$ and $\Gamma_{1}$ consists of straight lines. The basic equation $\left(2 \mathrm{a}^{*}\right)$ is reduced to the form

$$
\frac{1}{\rho(x, y)} \nabla^{2}\left[D(x, y) \nabla^{2} w_{0}(x, y)\right]-\lambda w_{0}(x, y)=0 .
$$

We now replace the boundary condition (4b) by the requirement:

$$
\int_{\Gamma_{2}}\left[D(x, y) p_{i}(x, y) \frac{\partial w_{0}(x, y)}{\partial n}\right] d s=0, \quad j=1,2,3, \cdots, m,
$$

where the functions $p_{i}(x, y)$ are selected to obey the following conditions:

(6a) The collection of functions $\left(D(x, y) p_{j}(x, y)\right), j=1,2,3, \cdots \infty$, forms a dense subset of $L_{2}\left[(\partial \Omega) \cap \Gamma_{2}\right]$.

(6b) Each function $\left(D(x, y) p_{i}(x, y)\right)$ is continuously differentiable on $\Gamma_{2}$.

(6c) Each function $\left(D(x, y) p_{i}(x, y)\right)$ is harmonic in $\Omega$.

(6d) Each function $p_{i}(x, y)$ is identically equal to zero on $\Gamma_{1}$.

Using (6b), (6c), (6d), (4a) and Green's theorem, we obtain:

$$
\begin{aligned}
\int_{\Gamma_{2}}\left(D p_{i} \frac{\partial w_{0}}{\partial n}\right) d s & =\int_{\partial \Omega}\left(D p_{i} \frac{\partial w_{0}}{\partial n}\right) d s \\
& =\int_{\partial \Omega}\left[D p_{i} \frac{\partial w_{0}}{\partial n}-w_{0} \frac{\partial}{\partial n}\left(D p_{i}\right)\right] d s \\
& =\left(D p_{i}, \nabla^{2} w_{0}\right)_{\Omega}-\left(\nabla^{2}\left(D p_{i}\right), w_{0}\right)_{\Omega} \\
& =\left(p j, D \nabla^{2} w_{0}\right)_{\Omega}=0
\end{aligned}
$$


which is now valid for $p_{1}, p_{2}, \cdots, p_{n}$. We observe that the equality (7) and Eq. (5) can be derived as a necessary condition for the weak extremum (see for example [18], page 21 for the definition) of the functional

$$
I\left(w_{0}\right)=\left(D \nabla^{2} w_{0}, \nabla^{2} w_{0}\right)_{\Omega}-\lambda\left(\rho w_{0}, w_{0}\right)_{\Omega}-2 \sum_{i=1}^{m} \mu i\left(p_{i}, D \nabla^{2} w_{0}\right)_{\Omega},
$$

where $\lambda, \mu_{i}$ are Lagrangian multipliers. By an elementary variational argument we can derive the necessary conditions for the extremum of $I\left(w_{0}\right)$ to be:

$$
\begin{gathered}
\frac{1}{\rho} \nabla^{2}\left(D \nabla^{2} w_{0}\right)-\lambda w_{0}=0 \quad \text { in } \Omega, \\
w_{0} \equiv \text { constant on } \partial \Omega, \\
\nabla^{2} w_{0}=\sum_{i=1}^{m} \mu_{i} p_{i} \quad \text { on } \partial \Omega .
\end{gathered}
$$

It follows from (9c) that $\nabla^{2} w_{0} \equiv 0$ on $\Gamma_{1}$, since all $p_{i}(x, y)$ vanish on $\Gamma_{1}$. Then our assumption that $\Gamma_{1}$ consists of straight lines implies that $\partial^{2} w_{0} / \partial n^{2} \equiv 0$ on $\Gamma_{1}$, since $w_{0} \equiv 0$ on $\Gamma_{1}, \partial^{2} w / \partial s^{2} \equiv 0$ on $\Gamma_{1}$, and $\nabla^{2} w_{0}=\left(\partial^{2} w / \partial s^{2}\right)+\left(\partial^{2} w / \partial n^{2}\right)$. (This would be false if $\Gamma_{1}$ had non-vanishing curvature.) Hence condition (3b) is automatically satisfied, and becomes a natural condition of the corresponding variational problem.

We introduce the "base problem," namely the problem of vibrating inhomogeneous membrane

$$
\begin{aligned}
\nabla^{2}\left(\frac{u(x, y)}{\rho(x, y)}\right) & =-\sqrt{ } \lambda u(x, y) & & \text { in } \quad \Omega, \\
u & \equiv 0 & & \text { on } \quad \partial \Omega .
\end{aligned}
$$

We assume that we know all we need to know about the "base problem." That is, ve know the eigenvalues $\lambda_{1}, \lambda_{2}, \cdots, \lambda_{m}$ and the corresponding eigenfunctions $\zeta_{1}$, ${ }_{3}, \cdots, \zeta_{n}$, which satisfy

$$
\begin{array}{cc}
\nabla^{2}\left(\zeta_{i} / \rho\right)=-\sqrt{\lambda_{i}} \zeta_{i} & \text { in } \quad \Omega, \\
\zeta_{i} \equiv 0 \quad \text { on } \quad \partial \Omega .
\end{array}
$$

Using the relationships (9) and Green's formula we have:

$$
\begin{aligned}
\left(D \nabla^{2} w_{0}, \zeta_{i}\right)_{\Omega}= & -\frac{1}{\sqrt{ } \lambda_{i}}\left(D \nabla^{2} w_{0}, \nabla^{2}\left(\zeta_{i} / \rho\right)\right)_{\Omega} \\
= & -\frac{1}{\sqrt{ } \lambda_{i}}\left\{\left(\nabla^{2}\left(D \nabla^{2} w_{0}\right), \frac{\zeta_{i}}{\rho}\right)_{\Omega}\right. \\
& \left.+\int_{\partial \Omega}\left[D \nabla^{2} w_{0} \cdot \frac{\partial}{\partial n}\left(\frac{\zeta_{i}}{\rho}\right)\right] d s-\int_{\partial \Omega}\left[\frac{\zeta_{i}}{\rho} \cdot \frac{\partial}{\partial n}\left(D \nabla^{2} w_{0}\right)\right] d s\right\} \\
= & -\frac{1}{\sqrt{ } \lambda_{i}}\left(\lambda \cdot \rho \cdot w_{0}, \frac{\zeta_{i}}{\rho}\right)_{\Omega}-\frac{1}{\sqrt{ } \lambda_{i}} \int_{\partial \Omega}\left[D \nabla^{2} w_{0} \cdot \frac{\partial}{\partial n}\left(\frac{\zeta_{i}}{\rho}\right)\right] d s \\
= & \frac{1}{\lambda_{i}}\left(\lambda w_{0}, \nabla^{2}\left(\frac{\zeta}{\rho}\right)\right)_{\Omega}-\frac{1}{\sqrt{ } \lambda_{i}} \int_{\partial \Omega}\left[D \nabla^{2} w_{0} \cdot \frac{\partial}{\partial n}\left(\frac{\zeta_{i}}{\rho}\right)\right] d s
\end{aligned}
$$




$$
\begin{aligned}
= & \frac{\lambda}{\lambda_{i}}\left\{\left(\nabla^{2} w_{0}, \frac{\zeta_{i}}{\rho}\right)_{\Omega}+\int_{\partial \Omega}\left(\frac{\zeta}{\rho} \cdot \frac{\partial w_{0}}{\partial n}\right) d s\right\} \\
& -\frac{1}{\sqrt{ } \lambda_{i}} \int_{\partial \Omega}\left[\sum_{i=1}^{m} \mu_{i} \cdot D \cdot p_{i} \cdot \frac{\partial}{\partial n}\left(\frac{\zeta_{i}}{\rho}\right)\right] d s \\
= & \frac{\lambda}{\lambda_{i}}\left(\nabla^{2} w_{0}, \frac{\zeta_{i}}{\rho}\right)_{\Omega}-\frac{1}{\sqrt{ } \lambda_{i}} \sum_{i=1}^{m}\left(D \cdot p_{i}, \nabla^{2}\left(\frac{\zeta_{i}}{\rho}\right)\right)_{\Omega} \\
= & \frac{\lambda}{\lambda_{i}}\left(\frac{\nabla^{2} w_{0}}{\rho}, \zeta_{i}\right)_{\Omega}+\sum_{i=1}^{m}\left(D p_{i}, \zeta_{i}\right)_{\Omega} .
\end{aligned}
$$

Comparing the first and last terms in our chain of equalities, we have

$$
\lambda_{i}\left(D \nabla^{2} w_{0}, \zeta_{i}\right)_{\Omega}=\lambda\left(\frac{D \nabla^{2} w_{0}}{D \cdot \rho}, \zeta_{i}\right)_{\Omega}+\lambda_{i} \sum_{i=1}^{m} \mu_{i}\left(D p_{i}, \zeta_{i}\right)_{\Omega}
$$

We now observe that the eigenfunctions $\zeta_{i}$ form orthogonal bases of the space $L_{2}(\Omega)$. The completeness follows from the completeness of the eigenfunctions of the Laplace's operator, and from the positive definite property of $\rho(x, y)$. The orthogonality is checked by considering for $\lambda_{i} \neq \lambda_{i}$ :

$\sqrt{ } \lambda_{i}\left(\zeta_{i}, \zeta_{i}\right)_{\Omega}-\sqrt{ } \lambda_{i}\left(\zeta_{i}, \zeta_{i}\right)_{\Omega}=\left(-\nabla^{2}\left(\zeta_{i} / \rho\right), \zeta_{i}\right)_{\Omega}$

Hence:

$$
+\left(\zeta_{i}, \nabla^{2}\left(\zeta_{i} / \rho\right)\right)_{\Omega}=\int_{\partial \Omega}\left[\zeta_{i} \cdot \frac{\partial}{\partial n}\left(\zeta_{i} / \rho\right)-\zeta_{i} \cdot \frac{\partial}{\partial n}\left(\zeta_{i} / \rho\right)\right] d s=0
$$

$$
\left(\left(\lambda_{i}-\frac{\lambda}{\rho D}\right) D \nabla^{2} w_{0}, \zeta_{i}\right)_{\Omega}=\lambda_{i} \sum_{i=1}^{m} \mu_{i}\left(D \cdot p_{i}, \zeta_{i}\right)_{\Omega}
$$

We observe that for an arbitrary $N$

$$
\sum_{i=1}^{N}\left(\left(\lambda_{i}-\frac{\lambda}{\rho D}\right) D \nabla^{2} w_{0}, \zeta_{i}\right) \zeta_{i}=\sum_{i=1}^{N}\left(\lambda_{i}-\frac{\lambda}{\rho D}\right) D \nabla^{2} w_{0}=\sum_{i=1}^{N} \lambda_{i} \sum_{i=1}^{m} \mu_{i}\left(D p_{i}, \zeta_{i}\right)_{\Omega} \cdot \zeta_{i}
$$

(assuming $\left\|\zeta_{i}\right\|=1$ without any loss of generality), and therefore

$$
D \nabla^{2} w_{0}=\sum_{i=1}^{\infty}\left\{\frac{\lambda_{i} \sum_{i=1}^{m} \mu_{i}\left(D p_{i}, \zeta_{i}\right) \zeta_{i}}{\lambda_{i}-\frac{\lambda}{\rho \cdot D}}\right\}
$$

with convergence of the right-hand side assured. But by Eq. (7) we have

Hence:

$$
\left(D \nabla^{2} w_{0}, p_{k}\right)_{\Omega}=0, \quad k=1,2, \cdots m .
$$

$$
\sum_{i=1}^{m} \mu_{i} \sum_{i=1}^{\infty} \lambda_{i}\left(D p_{i}, \zeta_{i}\right)_{\Omega}\left(\frac{\zeta_{i}}{\lambda_{i}-(\lambda / \rho D)}, p_{k}\right)_{a}=0, \quad k=1,2, \cdots, m .
$$

This can be regarded as a system of $m$ equations in $m$ unknowns, which can have a nontrivial solution only if the determinant of the coefficients is equal to zero:

$$
\operatorname{det}\left[\sum_{i=1}^{\infty} \lambda_{i}\left(D \cdot \zeta_{i}, p_{i}\right)_{\Omega}\left(\frac{D \zeta_{i}}{D \lambda_{i}-(\lambda / \rho)}, p_{k}\right)_{\Omega}\right]=0, \quad j, k=1,2, \cdots, m .
$$


This is the Weinstein determinant for our problem. In the case when $\rho$ and $D$ are constant it reduces to the result of $\mathrm{W}$ cinstein [2]:

$$
\operatorname{det} \sum_{i=1}^{\infty}\left\{\left(\rho \lambda_{i}\right) \cdot \frac{\left(\zeta_{i}, p_{i}\right)_{\Omega}\left(\zeta_{i}, p_{k}\right)_{\Omega}}{\left(\rho \lambda_{i}\right)-(\lambda / D)}\right\}=0, \quad j, k=1,2, \cdots, m .
$$

Since our $\left(\rho \lambda_{i}\right)$ corresponds to $\lambda_{i}$ of the Weinstein article, and our $\lambda / D$ to his $\lambda$, the formulas are clearly identical.

We note that changing to $r, \theta$ coordinates (cylindrical polars), a possible choice of the functions $p_{i}(\theta)$ for a starlike region $\Omega$ could be

$$
\begin{gathered}
\sin (n \theta), \cos (k \theta), \quad n=1,2, \cdots, k=0,1,2, \cdots, \text { on } \Gamma_{1}, \\
p_{i}(\theta) \equiv 0 \text { on } \Gamma_{2}, \quad p_{i}(\theta) \text { harmonic in } \Omega .
\end{gathered}
$$

This implies that we have to solve first Dirichlet's problem for the region $\Omega$. That is, however, easily done by well-known numerical procedures (for example, the relaxation technique). In a private communication Professor Weinstein has suggested to me a variant of a numerical computation for a region $\Omega$, such that a conformal map is known mapping $\Omega$ into the unit circle. Unfortunately, I did not attempt to follow his recipe.

I would like in this paragraph to comment finally on the unusual nature of the result obtained. The base problem considered in the usual Weinstein technique as originally given in [13] retains the differential operator and only changes the boundary conditions to construct a simpler boundary-value problem. In the papers of Bazley and Fox (see, for example, [4]) the differential operator is changed effecting the splitting of the operator into a sum of two operators, so that one of them turns out to be manageable, but then the boundary conditions remain unchanged.

Here we have replaced in the "base problem", the operator $(1 / \rho) \nabla^{2}\left(D \nabla^{2} \cdot\right)$, by the operator $\left(\left(\nabla^{2} / \rho\right) \cdot\right)$, but also the very complex boundary conditions assigned to the plate (specified separately on the subarcs of $\Gamma_{1}$ and $\Gamma_{2}$ ) were replaced by the very simple condition $u \equiv 0$ on $\partial \Omega$.

5. The general plate problem. If $\left.\diamond^{4}(I), w\right) \not \equiv 0$ in $\Omega$ the result of the preceding section does not apply. One may question the physical usefulness of our result since $D \neq$ constant, but $\diamond^{4}(D, w)=0$ imposes what seems to be an artificial variation of the thickness of the plate. However, this is exactly the condition which arises in the optimum weight-strength design of thin plates. (Given a maximum permitted stress level, we wish to design a plate of minimum weight not exceeding the prescribed maximum stress level.)

For this reason the simpler form of the plate equation:

$$
(1 / \rho) \nabla^{2}\left(D \nabla^{2} w\right)+\left(\partial^{2} w / \partial t^{2}\right)=0, \quad D(x, y) \not \equiv \text { const. }
$$

should be studied for other reasons than pure academic curiosity. In the general case, as given by Eq. (1), the variational problem has to be modified and we seek instead an extremum of the functional

$$
L\left(w_{0}\right)=\left(D \nabla^{2} w_{0}, \nabla^{2} w_{0}\right)_{\Omega}-(1-\nu)\left(D, \diamond^{4}\left(w_{0}, w_{0}\right)\right)_{\Omega}-\lambda\left(\rho w_{0}, w_{0}\right)_{\Omega}
$$

subject to the same type of a boundary constraint:

$$
w \equiv 0 \quad \text { on } \quad \partial \Omega,
$$




$$
\left(D p_{i}, \frac{\partial w}{\partial n}\right)_{\Gamma_{2}}=0, \quad j=1,2, \cdots, m .
$$

There are some subtle difficulties in handling the product $\left(D, \nabla^{4}(w, w)\right)_{\Omega}$ which have prevented what seems like an obvious extension of the result of this paper.

\section{REFERENCES}

[1] N. Aronszajn, The Raleigh-Ritz method and A. Weinstein method for approximation of eigenvalues, $I$, Proc. Nat. Acad. Sci. U. S. A. 34 (1948), 474-480

[2] —, II, Proc. Nat. Acad. Sci. U. S. A. 34 (1948), 594-601

[3] —-, Approximation methods for eigenvalues of completely continuous symmetric operators, in Proc. Symp. Spectral Theory and Differential Problems, Oklahoma State Univ., Stillwater, Okla., 1951

[4] N. Bazley and D. W. Fox, Truncation in the method of intermediate problems for lower bounds to eigenvalues, J. Res. Nat. Bur. Stds. 65B, (1961)

[5] - Methods for lower bounds to frequencies of contimuous elastic systems, John Hopkins Univ. Applied Physics Lab. Report TG 609, 1964

[6] N. Bazley, Lower bounds for eigenvalues, J. Math. Mech. 10 (1961), 289-308

[7] J. B. Diaz, Upper and lower bounds on eigenvalues, in 8th Symposium in Applied Mathematics, A. M. S., pp. 53-58, New York, 1958

[8] G. Fichera, Linear elliptic differential systems and eigenvalue problems, Lect ure Notes in Mathematics, Springer-Yerl ag, 1964

[9] — Lezioni sulle transformazioni lineari, Inst. Math. Univ. Trieste, 1954.

[10] S. H. Gould, Variational methods in eigenvalue problems, Univ. of Toronto Press, Toronto 1957

[11] T. Kato, Quadratic forms in Hilbert space and asymptotic perturbation series, Univ. of California, lecture notes, Berkeley, Calif., 1955

[12] L. E. Payne, Inequalities for eigenvalues of membranes and plates, J. Rat. Mech. Anal. 4 (1955), $517-529$

[13] A. Weinstein, Sur la stabilité des plaques encastrées, Compt. Rend. 200 (1935), 107-109

[14] - Intermediate problem and the maximum-minimum theory of eigenvalues, J. Math. Mech. 12, 235-246, (1963)

[15] — Some applications of the new maximum-minimum theory of eigenvalues, J. Math. Anal. Applic. 12 (1965), 58-64

[16] - A necessary and sufficient condition in the maximum-minimum theory of eigenvalues, studies in mathematical analysis and related topics. Stanford, Stanford Univ. Press, 1962

[17] - Bounds for eigenvalues and the method of intermediate problems, in proceedings of the international conference on partial differential equations and continuum mechanics, Madison, University of Wisconsin Press, 1961, pp. 39-53.

[18] L. E. Elsgolc, Calculus of variations, Addison-Wesley, Reading Mass., 1962

[19] S. T. Kuroda, Finite dimensional perturbation and representation of the scattering operator, Pacific J. of Math. 13 (1963), 1305-1318 